\title{
ELECTRICALLY CONDUCTING FLOW THROUGH EXPONENTIAL POWER LAW FLUID WITH VARIABLE THERMAL CONDUCTIVITY
}

\author{
M. FERDOWS ${ }^{*}$ and M.Z.I. BANGALEE \\ Research Group of Fluid Flow Modeling and Simulation \\ Department of Applied Mathematics, University of Dhaka \\ Dhaka-1000, BANGLADESH \\ E-mail: ferdows@du.ac.bd
}

D. LIU

College of Engineering and Science, Louisiana Tech University

Ruston, Louisiana, 71272, USA

\begin{abstract}
The problem of exponential law of steady, incompressible fluid flow in boundary layer and heat transfer are studied in an electrically conducting fluid over a semi-infinite vertical plate assuming the variable thermal conductivity in the presence of a uniform magnetic field. The governing system of equations including the continuity equation, momentum equation and energy equation have been transformed into nonlinear coupled ordinary differential equations using appropriate similarity variables. All the numerical and graphical solutions are obtained through the use of Maple software. The solutions are found to be dependent on three dimensionless parameters including the magnetic field parameter $M$, thermal conductivity parameter $\beta$ and Prandtl number Pr. Representative velocity and temperature profiles are presented at various values of the governing parameters. The skin-friction coefficient and the rate of heat transfer are also calculated for different values of the parameters.
\end{abstract}

Key words: MHD, similarity solution, variable properties, boundary layer flow, exponential law.

\section{Introduction}

Magneto-hydrodynamics (MHD) deals with the study of electrically conducting fluids and magnetism. At the view of MHD magnetic field induces current in a fluid and plays an important role in velocity and temperature of the fluid. There is a wide variety of situations where MHD problem arises from the origin of the Earth's magnetic field to the prediction of weather, the damping of turbulent fluctuations in semiconductor melts during crystal growth and even in the measurement of the flow rates of beverages in food industry. An interesting application of MHD to metallurgy lies in purification of molten metal from non-metallic inclusions by the application of a transverse magnetic field. In recent years MHD problems have become more important industrially. Indeed, exponential law of fluid is a significant type of behavior having considerable practical applications in chemical engineering, electrochemistry and polymer processing in many natural phenomena and scientific problems.

Soundalgekar and Takhar [1] solved the modified Blasius-type of boundary layer equations for velocity and temperature field without neglecting the heat due to stress work. It was observed that the magnetic field does create stress in the fluid thereby causing the generation of heat. The induced magnetic field was assumed to be negligible. Rossow [2] studied the effects of the transverse magnetic field on the flow and heat transfer of an electrically conducting viscous fluid past a semi-infinite flat plate. He assumed that the induced magnetic field is very small and hence negligible. Takhar et al. [3] investigated the forced and free convective flow past a semi-infinite vertical plate taking account of viscous dissipative heat.

\footnotetext{
* To whom correspondence should be addressed
} 
Sakiadis [6] studied the boundary layers on a continuous semi-infinite sheet moving steadily through a quiescent fluid environment. Kumari et al. [7] analyzed the MHD flow and heat transfer over a stretching surface with prescribed wall temperature or heat flux. A non-similar, laminar, steady, electrically conducting forced convection liquid metal boundary flow with induced magnetic field effects was studied by Beg et al. [8]. Takhar [9] considered aligned magnetic field effects on laminar hydrodynamic boundary layer convection along an impulsively-started semi-infinite plate. Srinivasa and Eswara [10] studied an unsteady laminar boundary layer flow due to an impulsively stretching surface. Devi and Nagraj [11] investigated heat and mass transfer in an unsteady magneto hydrodynamic flow over a semi-infinite flat plate. Elbashbeshy [12] considered heat transfer over an unsteady stretching surface with variable heat flux in the presence of heat source or sink.

Chamkha [13] studied the problem of steady, laminar, free convection flow over a vertical porous surface in the presence of magnetic field. Watanabe [14] numerically analyzed the natural convection hydrodynamic wedge flow in the presence of a transverse magnetic field.

The steady, laminar boundary layer, two-dimensional magnetohydrodynamic (MHD) flow past a continuously moving semi-infinite vertical porous plate taking into account the Dufour and Soret effects (double diffusion) on variation of fluid viscosity with temperature was studied by Ferdows et al. [15]. Idowu et al. [16] studied the heat and mass transfer of MHD and dissipative fluid flow past a moving vertical porous plate with variable suction. Sarveshanand and Singh [17] investigated the steady two-dimensional hydromagnetic free convective flow of an incompressible viscous and electrically conducting fluid between two parallel vertical porous plates. Recently, an exact solution for fully developed MHD viscous flow of an electrically conducting fluid in a vertical micro-channel in the presence of velocity slip and temperature jump at the micro-channel walls was studied by Jha and Aina. The present paper extends the previous works to study the exponential law of velocity of a fluid for the boundary layer problem in the presence of a uniform magnetic field.

\section{Problem formulation}

A steady exponentially convective boundary layer flow adjacent to a heated vertical plate is considered at a constant wall temperature $T_{w}$ whereas the uniform ambient temperature is $T_{\infty}$. Here the $y$-axis is taken along a semi-infinite plate in the direction of flow and the $x$-axis is taken normal to it. A transverse magnetic field of uniform strength is assumed to be applied to the plate. Let the magnitude of the transverse magnetic field be $\left(0, B_{0}, 0\right)$. The boundary layer approximations of an electrically conducting fluid are the mass conservation, the momentum and the energy equation

$$
\begin{aligned}
& \frac{\partial u}{\partial x}+\frac{\partial v}{\partial y}=0, \\
& u \frac{\partial u}{\partial x}+v \frac{\partial u}{\partial y}=v \frac{\partial^{2} u}{\partial y^{2}}+\frac{\sigma B_{0}^{2}}{\rho}\left(U_{\infty}^{2}-u^{2}\right), \\
& u \frac{\partial T}{\partial x}+v \frac{\partial T}{\partial y}=\alpha \frac{\partial^{2} T}{\partial y^{2}} .
\end{aligned}
$$

Subject to the boundary conditions

$$
u(0)=0 ; v(0)=0 ; T(0)=T_{W} ; u(\infty)=U_{\infty} ; T(\infty)=T_{\infty}
$$

where $u$ and $v$ are the components of velocity in the $x$ and $y$ directions; $T$ is the temperature; $\sigma, \rho$ and $v$ are the electrical conductivity, density and kinematic viscosity respectively, $B_{0}$ is the magnetic induction and $\alpha$ is the thermal diffusivity with $U_{\infty}$ being the free stream velocity. 
In order to facilitate the analysis of a full set of governing equations, along with the boundary conditions Eqs (2.1)-(2.3) are dimensionless and the following similarity variables are introduced [3]

$$
\begin{aligned}
& \eta=y(\exp (m x / 4)) C, \\
& \psi=\left(U_{0} / C\right) \exp (m x / 4) f(\eta), \\
& \theta=\frac{T-T_{\infty}}{T_{W}-T_{\infty}}, \\
& U_{\infty}=U_{0} \exp \left(\frac{m x}{2}\right), \\
& U_{0}=U_{\infty} \quad \text { at } \quad x=0, \quad C=\left(\frac{m U_{0}}{4 v}\right)^{1 / 2}
\end{aligned}
$$

where $\eta$ is the dimensionless similarity variable and $m$ is a positive constant. The thermal diffusivity is taken as usual as in [5]

$$
k=k_{0}\left[1+\alpha\left(T-T_{\infty}\right)\right]=k_{0}[1+\beta \theta]
$$

where $\beta=\alpha\left(T_{W}-T_{\infty}\right)$ is the thermal conductivity parameter.

Substituting the similarity variables into the governing Eqs (2.1) to (2.3), we obtain the equations

$$
\begin{aligned}
& 2\left(f^{\prime}\right)^{2}-f f^{\prime \prime}=f^{\prime \prime \prime}+4 M\left(1-\left(f^{\prime}\right)^{2}\right), \\
& (1+\beta \theta) \theta^{\prime \prime}+\beta \theta^{\prime 2}+\operatorname{Pr} f \theta^{\prime}=0
\end{aligned}
$$

where the prime(s) indicates the differentiation with respect to $\eta$.

The boundary conditions (2.4) are

$$
\begin{aligned}
& f=f^{\prime}=0, \quad \theta=1 \quad \text { at } \quad \eta=0 ; \\
& f^{\prime} \rightarrow 1, \quad \theta \rightarrow 0 \quad \text { as } \quad \eta \rightarrow \infty .
\end{aligned}
$$

The dimensionless parameters are the magnetic parameter $M=\frac{\sigma B_{0}^{2}}{m \rho}$, Prandtl number, $\operatorname{Pr}=\frac{v}{\alpha}$ and thermal conductivity parameter $\beta$.

\section{Numerical analysis and results}

The system of governing Eqs (2.7), (2.8) with the transformed boundary conditions (2.9) are solved using Maple [4] for a range of the magnetic field parameter $M=1,2,3.8$, thermal conductivity parameter $\beta=0,0.7,1.3$ chosen arbitrarily and Prandtl number $\operatorname{Pr}=0.71,5,10$ representing air, water and oil respectively in order to see the flow profiles of velocity and temperature and also the values of the skin friction coefficient and rate of heat transfer near the wall. 
In Figs 1 and 2, respectively, we see the behavior of velocity $\left(f^{\prime}(\eta)\right)$ and temperature $(\theta(\eta))$ profiles as a function of similarity variables $\eta$ for different values of the Prandtl number Pr. Considering the magnetic field it is seen that the presence of Pr does not cause any change to the fluid velocity. From the governing equations we can easily find that $f^{\prime}$ depends only on $M$ not on Pr. It is evident from the simulation that the boundary conditions $f^{\prime}(0)=0$ and $f^{\prime}(\infty)=1$ are satisfied.

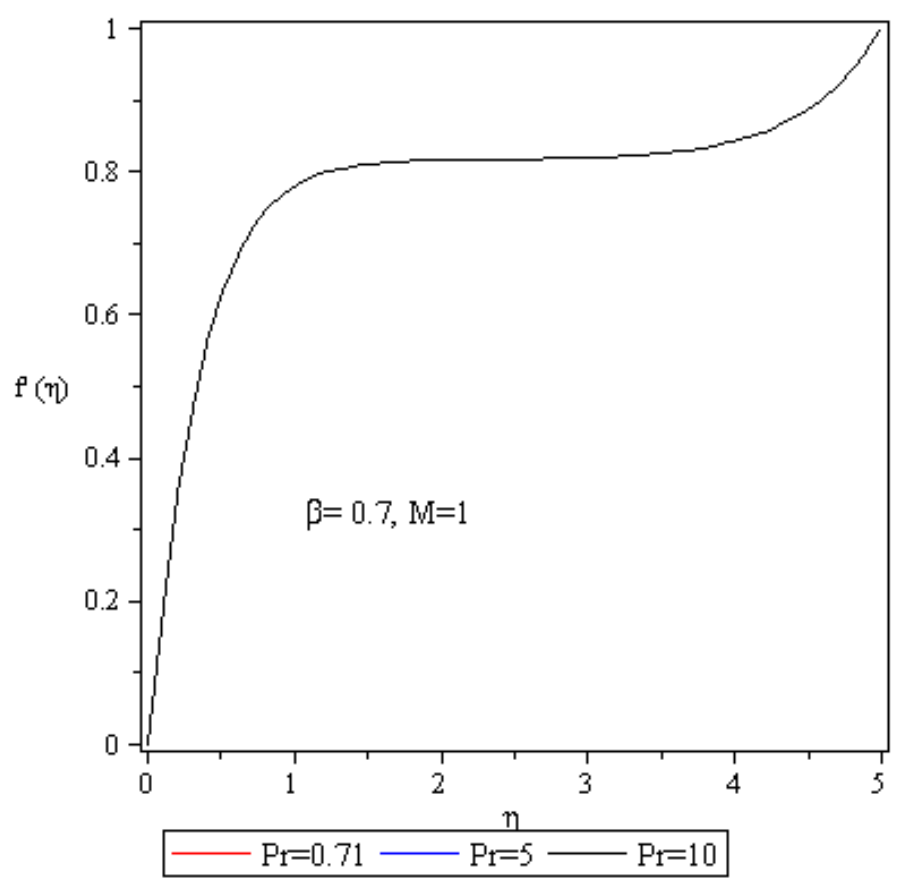

Fig.1. Velocity profiles for various Pr.

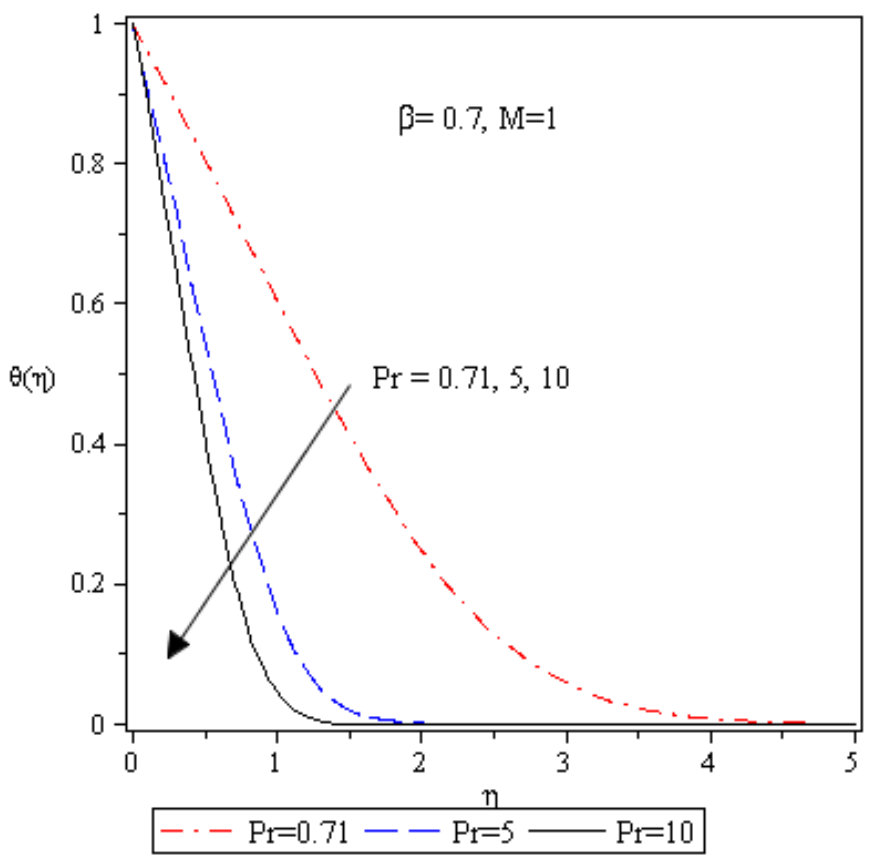

Fig.2. Temperature profile for various Pr. 
The temperature profile depends on the value of the Prandtl number and the impact is shown in Fig.2. We can see that the thermal boundary layer thickness is reduced as Pr increases from air to oil. The variation of temperature profiles with the thermal conductivity parameter is depicted in Fig.3. We can see the fact that the thermal boundary layer induced more flow at higher $\beta$. Figures 4 and 5 show the effect of magnetic field parameter $M$ on velocity and temperature profiles. It is observed that the effect of increasing magnetic parameter increases the velocity and decreases the temperature.

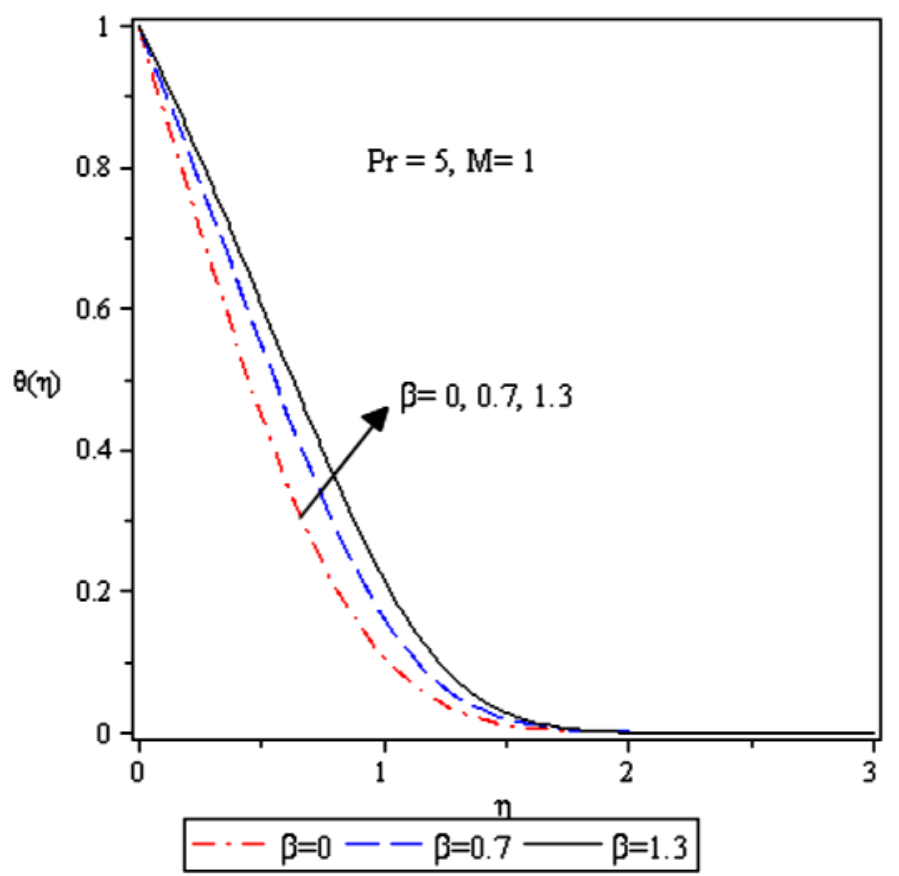

Fig.3. Temperature profile for various values of $\beta$.

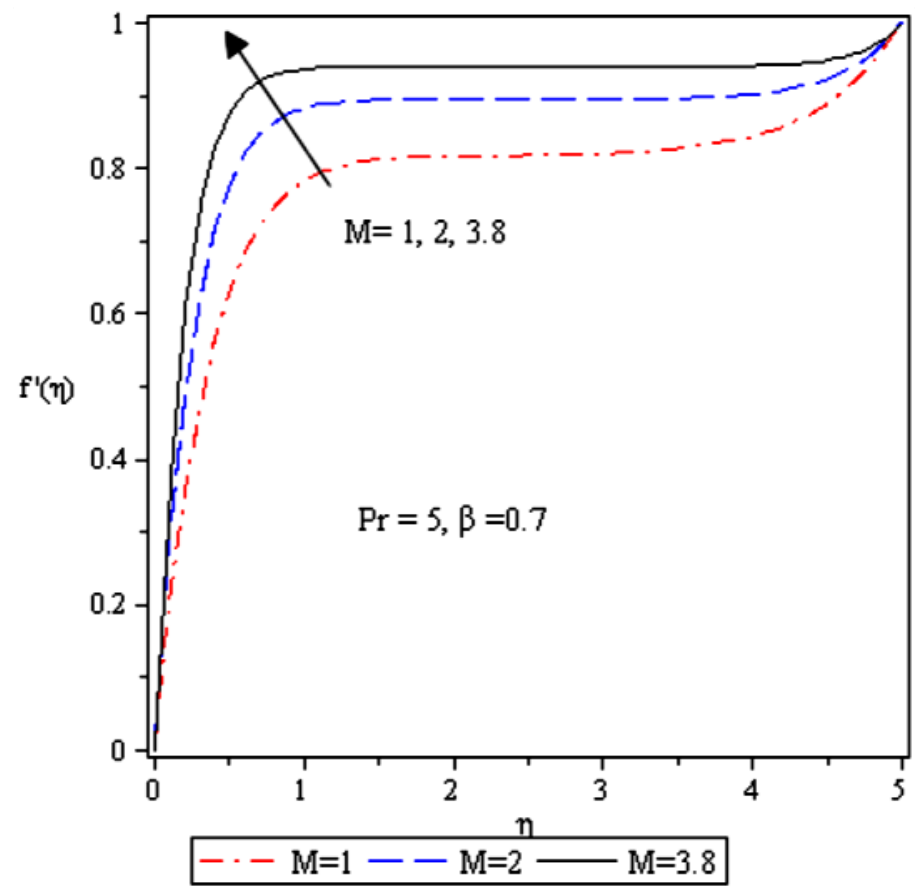

Fig.4. Velocity profiles for various $M$. 


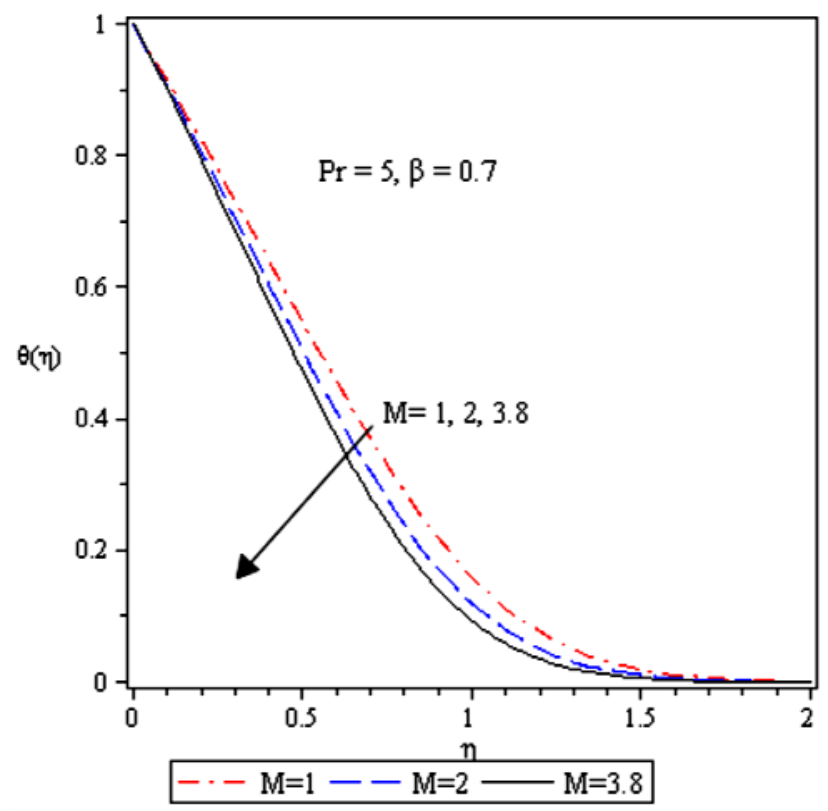

Fig.5. Temperature profiles for various $M$.

The dimensionless skin-friction coefficient and rate of heat transfer coefficient are given by

$$
\begin{aligned}
& \tau=\mu C U_{0} \exp \left(\frac{3 m x}{4}\right) f^{\prime \prime}(0), \\
& q=-k C\left(T_{W}-T_{\infty}\right) \exp \left(\frac{m x}{4}\right) \theta^{\prime}(0) .
\end{aligned}
$$

\begin{tabular}{|c|c|c|c|}
\hline Parameters & Values & $f^{\prime \prime}(0)$ & $-\theta^{\prime}(0)$ \\
\hline \multirow{6}{*}{$M$} & 1 & 2.11444164669409 & 0.844877152292886 \\
\hline & 2 & 3.10935734917004 & 0.925195016421425 \\
\hline & 3.8 & 4.38134066190016 & 0.992422270859739 \\
\hline & 5 & 5.05724712382262 & 1.01839078338458 \\
\hline & 7 & 6.01858143464882 & 1.04781072093380 \\
\hline & 10 & 7.22554774103531 & 1.07606564682467 \\
\hline \multirow{6}{*}{$\beta$} & 0 & 2.10993016740582 & 1.14955324186280 \\
\hline & 0.7 & 2.10993043948920 & 0.842346324691074 \\
\hline & 1.3 & 2.10993043946544 & 0.715333633060707 \\
\hline & 1.5 & 2.10993043945988 & 0.684780505765151 \\
\hline & 2 & 2.10993043994654 & 0.623602651144721 \\
\hline & 2.5 & 2.10993044010258 & 0.577179199239087 \\
\hline \multirow{8}{*}{$\operatorname{Pr}$} & 0.71 & 2.10950107252065 & 0.372419758936050 \\
\hline & 1.5 & 2.10950100374000238 & 0.514016938760667 \\
\hline & 2.5 & 2.10950100979246 & 0.636260164898946 \\
\hline & 5 & 2.10950100544791 & 0.842150024263920 \\
\hline & 7 & 2.10950108491696 & 0.961456966411895 \\
\hline & 10 & 2.10950108490393 & 1.10382103341652 \\
\hline & 13 & 2.10950108491696 & 1.22006982142798 \\
\hline & 15 & 2.10950108492492 & 1.28793534880790 \\
\hline
\end{tabular}

Table 1. Values of $f^{\prime \prime}(0)$ and $-\theta^{\prime}(0)$. 
The variation of the dimensionless shear stress and rate of heat transfer with varying $M$ is illustrated in Figs 6 and 7.

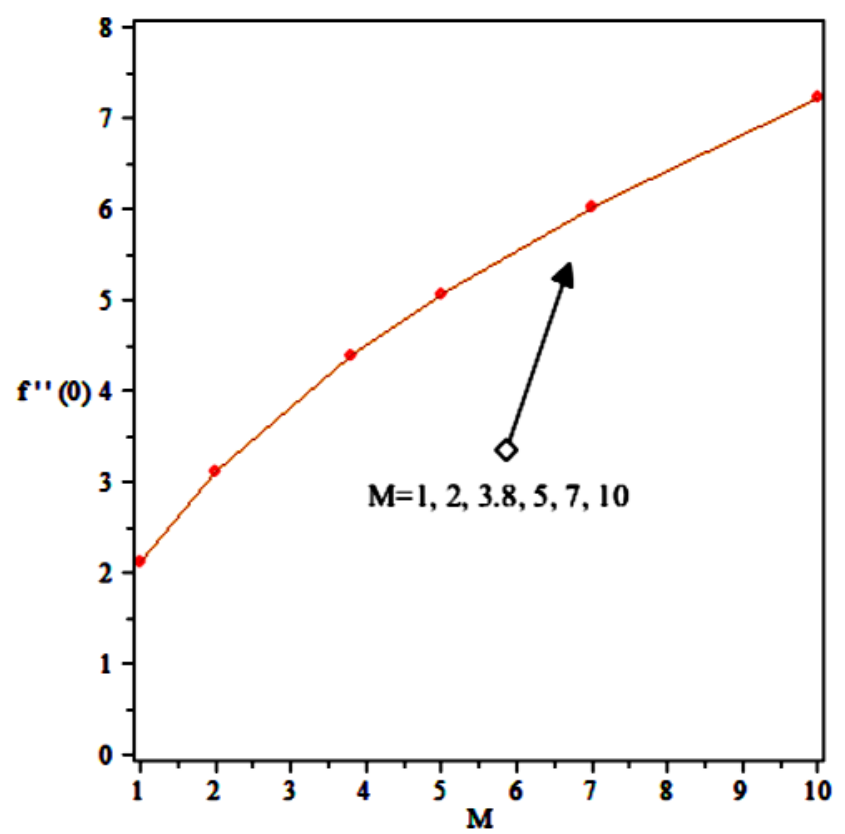

Fig.6. Skin-friction due to $M$.

Figure 6 leads us to conclude that the shearing stress increases with increasing $M$. We observe that the shearing stress remains constant for various values of $\beta$ and Pr.

On the other hand, we found that $-\theta^{\prime}(0)$ is increasing monotonically as $M$ increases as shown in Fig.7. Further, we can notice from Figs 8 and 9 that $-\theta^{\prime}(\theta)$ is decreasing as $\beta$ increases and on the other hand, $-\theta^{\prime}(0)$ increases with the increase of the parameter Pr.

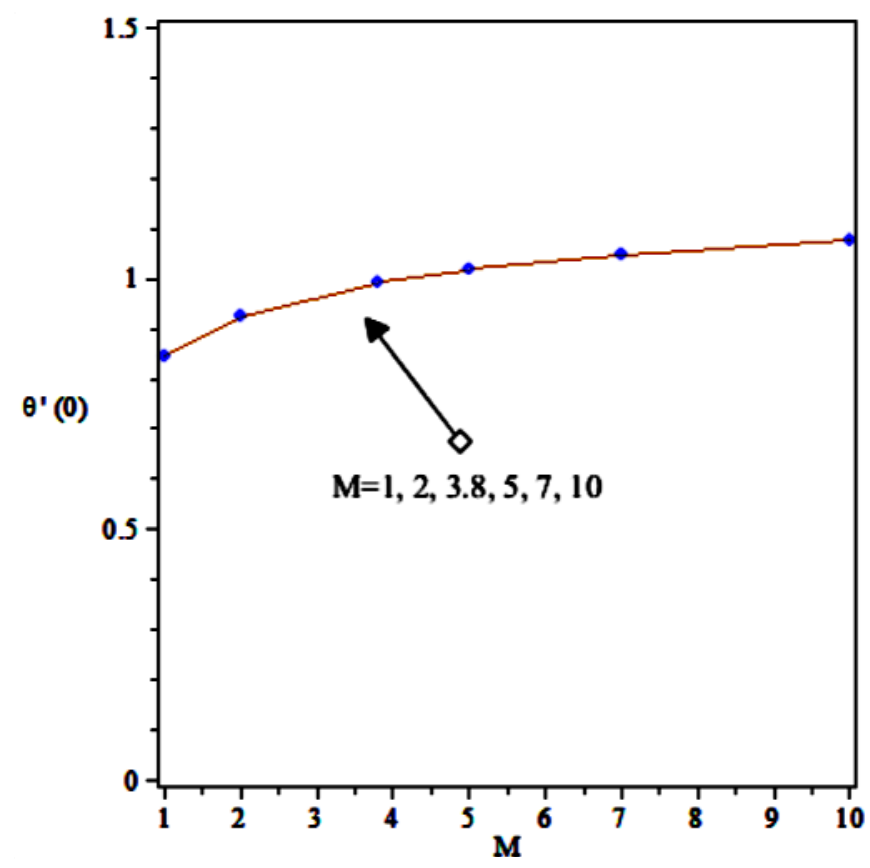

Fig.7. Rate of heat transfer due to $M$. 


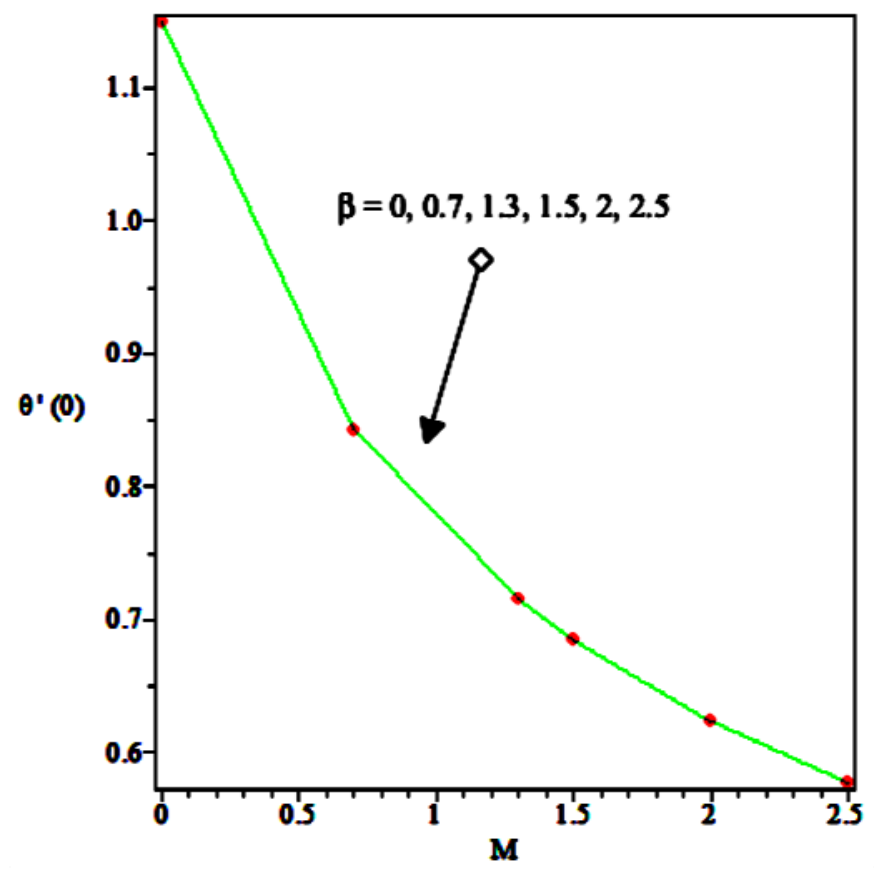

Fig.8. Rate of heat transfer due to $\beta$.

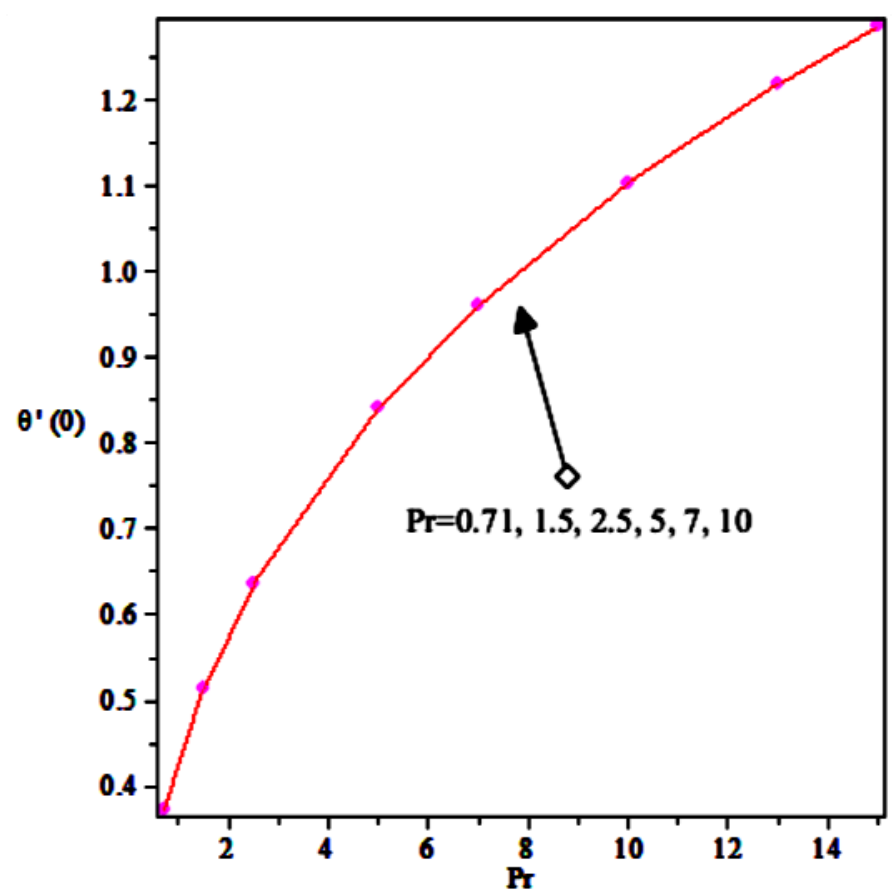

Fig.9. Rate of heat transfer due to Pr.

\section{Conclusion}

The most significant results are as follows:

(1) The velocity profiles depend on the magnetic field parameter, $M$ only.

(2) Temperature profile depends on all the dimensionless parameters $M, \operatorname{Pr}$ and $\beta$.

(3) For increasing values of $M$, the values of $f^{\prime \prime}(0)$ gradually increase. Thus the skin-friction coefficient increases with the increase of the magnetic field parameter $M$. 
(4) $-\theta^{\prime}(0)$ decreases as $M$ increases. Hence the rate of heat transfer at the wall increases.

(5) The rate of heat transfer decreases as parameter $\beta$ increases. However, heat transfer increases with the increase of the Prandtl number Pr.

(6) Since the velocity profile only depends on the parameter $M$, the skin-friction is constant with the change of other two dimensionless parameters $\beta$ and $\mathrm{Pr}$.

\section{Nomenclature}

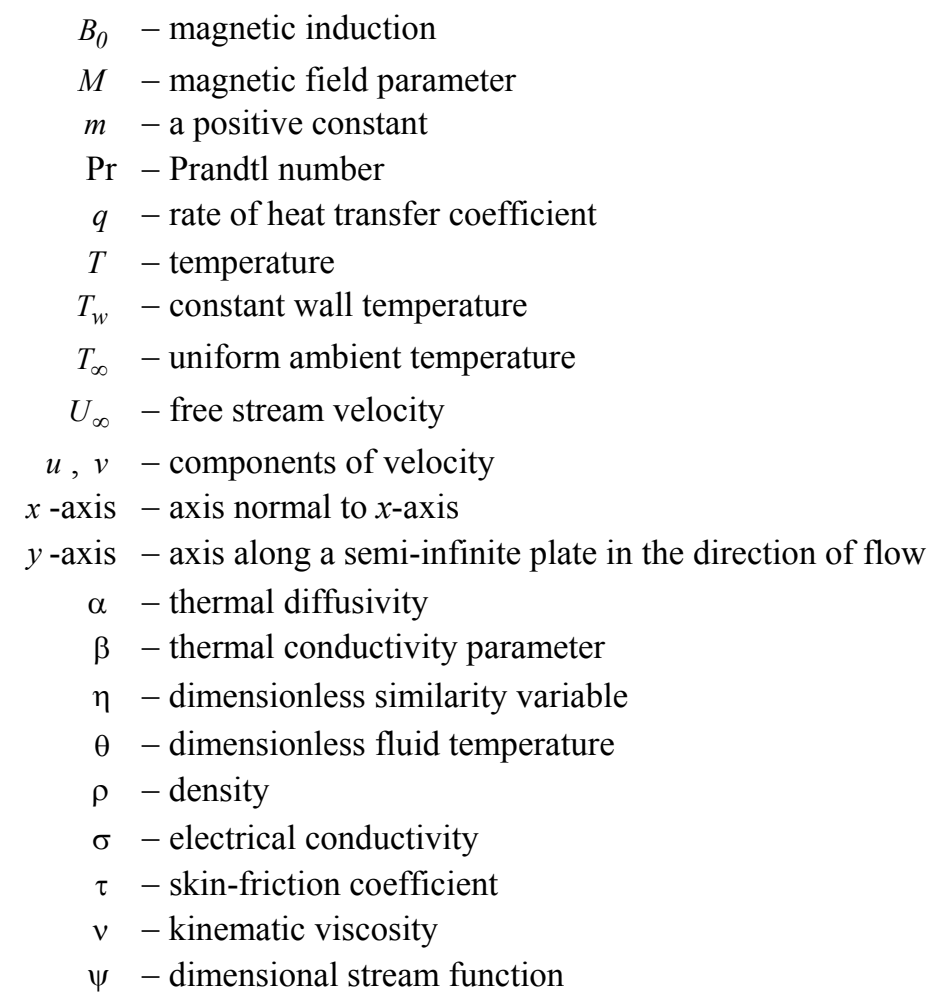

\section{References}

[1] Soundalgekar V.M. and Takhar H.S. (1977): On MHD flow and heat transfer over a semi-infinite plate under the transverse magnetic field. - Nuclear Engineering and Design, vol.42, pp.233-236.

[2] Rossow V.J. (1958): On flow of electrically conducting fluids over a flat plate in the presence of a transverse magnetic field. - NACA, Rept. No.1358.

[3] Takhar H.S., Byrne J.E. and Soundalgekar V.M (1976): Viscous dissipation effects on combined forced and free convection in a boundary layer flow. - MECH. RES. COMM, vol.3, pp.451-455.

[4] Aziz A. (2006): Heat Conduction with Maple. - USA: Edwards Inc.

[5] Slattery J.C. (1972): Momentum, Energy and Mass Transfer in Continuum. - New York: MC Graw-Hill.

[6] Sakiadis B.C. (1961): Boundary layer behavior on continuous solid surface. - AIChEJ, vol.7, pp.26-28.

[7] Kumari M., Takhar H.S. and Nath G. (1990): MHD flow and heat transfer over a stretching surface with prescribed wall temperature or heat flux. - Warme and Stoffubertragung, vol.25, pp.331-336. 
[8] Beg A., Bakir A.Y., Prasad V.R. and Ghosh S.K. (2009): Nonsimilar, laminar, steady, electrically conducting forced convection liquid metal boundary layer flow with induced magnetic field effects. - International Journal of Thermal Science, vol.48, pp.1596-1606.

[9] Takhar H.S. (1999): Unsteady flow and heat transfer on a semi-infinite flat plate with an aligned magnetic field. Int. J. Engineering Science, vol.37, No.13, pp.1723-1736.

[10] Srinivasa A.H. and Eswara A.T. (2011): Unsteady MHD Laminar Boundary Layer due to an impulsive stretching surface. - World Congress on Engineering (WCE), vol.1, pp.252-255.

[11] Sarma Devi C.D. and Nagraj M. (1984): Heat and mass transfer in unsteady MHD flow over a semi infinite flat plate. - Indian J. Pure App. Math, vol.15, No.10, pp.1148-1161.

[12] Elbashbeshy E.M.A. and Aldawody D. (2010): Heat transfer over an unsteady stretching surface with variable heat flux in the presence of heat source or sink. - Journal Computer and Mathematics with Applications, vol.60, No.10, pp.2806-2811.

[13] Chamkha A.J. (1999): Steady, laminar, free convection flow over a wedge in the presence of a magnetic field and heat generation or absorption. - Int. J. Heat and Fluid Flow, vol.20, No.84.

[14] Watanabe T. (1993): MHD free convection flow over a wedge in the presence of a transverse magnetic field. - Int. Comm., Heat and Mass Transfer, vol.20, pp.471-480.

[15] Ferdows M., Bangalee M.Z.I., Crepeau J.C. and Seddeek M.A. (2011): The effect of variable viscosity in double diffusion problem of MHD from a porous boundary with internal heat generation. - Progress in Computational Fluid Dynamics, An International Journal, vol.11, No.1, pp.54-65.

[16] Idowu A.S., Dada M.S. and Jimoh A. (2013): Heat and mass transfer of magnetohydrodynamic (MHD) and dissipative fluid flow past a moving vertical porous plate with variable suction. - Mathematical Theory and Modeling, vol.3, No.3, pp.80-110.

[17] Sarveshanand and Singh A.K. (2015): Magnetohydrodynamic free convection between vertical parallel porous plates in the presence of induced magnetic field. - Springerplus, vol.4, No.333.

[18] Jha B. and Babatunde A. (2018): Interplay of non-conducting and conducting walls on magnetohydrodynamic (MHD) natural convection flow in vertical micro-channel in the presence of induced magnetic field. - Propulsion and Power Research, 2018, Article in Press.

Received: April 21, 2018

Revised: December 16, 2018 